The Astrophysical Journal, 653:700-707, 2006 December 10

(C) 2006. The American Astronomical Society. All rights reserved. Printed in U.S.A.

\title{
ON THE SEARCH FOR TRANSITS OF THE PLANETS ORBITING GLIESE 876
}

\author{
P. D. Shankland, ${ }^{1,2}$ E. J. Rivera,${ }^{3}$ G. Laughlin, ${ }^{3}$ D. L. Blank, ${ }^{1}$ A. Price, ${ }^{4}$ B. Gary, ${ }^{4}$ R. Bissinger,${ }^{4}$ F. Ringwald ${ }^{5}$ \\ G. White, ${ }^{1}$ G. W. Henry, ${ }^{6}$ P. McGee, ${ }^{7}$ A. S. Wolf, ${ }^{3}$ B. Carter, ${ }^{8}$ S. Lee, ${ }^{9}$ J. Biggs, ${ }^{10}$ \\ B. Monard, ${ }^{11}$ and M. C. B. Ashley ${ }^{12}$ \\ Received 2006 May 7; accepted 2006 August 21
}

\begin{abstract}
We report the results of a globally coordinated photometric campaign to search for transits by the $P \sim 30$ day and $P \sim 60$ day outer planets of the three-planet system orbiting the nearby M dwarf Gl 876. These two planets experience strong mutual perturbations, which necessitate the use of a dynamical (four-body) model to compute transit ephemerides for the system. Our photometric data have been collected from published archival sources, as well as from our photometric campaigns that were targeted to specific transit predictions. Our analysis indicates that transits by planet $\mathrm{c}$ $(P \sim 30$ days $)$ do not currently occur, in concordance with the best-fit $i=50^{\circ}$ coplanar configuration obtained by dynamical fits to the most recent radial velocity data for the system. Transits by planet $\mathrm{b}(P \sim 60$ day $)$ are not entirely ruled out by our observations, but our data indicate that it is very unlikely that they occur. Our experience with the Gl 876 system suggests that a distributed ground-based network of small telescopes can be used to search for transits of very low mass $\mathrm{M}$ stars by terrestrial-sized planets.
\end{abstract}

Subject headings: planetary systems — planets and satellites: general — stars: individual (Gliese 876)

\section{INTRODUCTION}

The G1 876 planetary system ranks with the most remarkable discoveries that have emerged from the first decade of extrasolar planet detections. As described by Rivera et al. (2005), Laughlin et al. (2005), Butler et al. (2001), Marcy et al. (1998, 2001), and Delfosse et al. (1998), there are a number of reasons why the Gl 876 planets are extraordinary. Planet d with its 1.974 day orbit and $M \sin (i)=5.9 M_{\oplus}$, is the lowest mass exoplanet known to orbit a nearby main-sequence star (Rivera et al. 2005). Planets c and $b$ are the only Jovian-mass companions known to orbit an $\mathrm{M}$ dwarf, and their clear participation in 2:1 mean-motion resonance gives important dynamical clues to the formation and evolution of the system (e.g., Lee \& Peale 2002). Furthermore, the Gl 876 planets are the closest exoplanets that have been reliably characterized. Their radial velocity amplitudes induce an overall signal-to-noise ratio of nearly 100 , and they have been observed for well over a decade.

\footnotetext{
1 Centre for Astronomy, School of Mathematical and Physical Sciences, James Cook University, Townsville QLD 4811, Australia.

2 Current address: US Naval Observatory, 3450 Massachusetts Avenue NW, Washington, DC 20392-5420; paul.shankland@usno.navy.mil.

${ }^{3}$ University of California/Lick Observatory, University of California at Santa Cruz, Santa Cruz, CA 95064.

4 American Association of Variable Star Observers, Clinton B. Ford Astronomical Data and Research Center, 25 Birch St., Cambridge, MA 02138.

5 Department of Physics, California State University, Fresno, 2345 East San Ramon Avenue, M/S MH37, Fresno, CA 93740.

6 Center of Excellence in Information Systems, Tennessee State University, 3500 John A. Merritt Boulevard, Box 9501, Nashville, TN 37209.

7 School of Chemistry and Physics, University of Adelaide, Adelaide SA 5005, Australia.

${ }^{8}$ Centre for Astronomy, Solar Radiation and Climate Physics, University of Southern Queensland, Towoomba 4350, Australia.

9 Anglo-Australian Observatory, 167 Vimiera Road, Eastwood NSW 2122, Australia.

${ }^{10}$ Perth Observatory, Walnut Road, Bickley WA 6076, Australia.

11 Bronberg Observatory, Centurion 0157, Pretoria, South Africa

12 School of Physics, University of New South Wales, Sydney, NSW 2052, Australia.
}

The detection of Gl $876 \mathrm{~d}$ by Rivera et al. (2005) underscored the rapid development of the Doppler technique. The detection of planets having only a few Earth masses is the culmination of advances described by Butler et al. (1996), Marcy \& Butler (1998), Marcy et al. (2000, 2005), and Lovis et al. (2006). Nevertheless, while radial velocity precision has improved to better than $1 \mathrm{~m} \mathrm{~s}^{-1}$, Keplerian orbital fits to Doppler velocities give estimates of $M_{\mathrm{pl}} \sin (i)$ rather than $M_{\mathrm{pl}}$. In order to ascertain the true mass of a planet, one must obtain an independent measure of the orbital inclination $i$, which is most easily measured when the planet is observed to transit across the face of the parent star.

The now celebrated companion to HD 209458 provided the first example of a transiting extrasolar planet, and this body has generated an incredible variety of observational and theoretical investigations. HD 209458 b was initially detected with the radial velocity method, and was then discovered to transit on the basis of follow-up photometric observations at the predicted transit times (Henry et al. 1999, 2000; Charbonneau et al. 2000). HD 209458b's well-sampled light curve, when combined with high-precision radial velocity data, has permitted precise measurements of the planet's basic attributes (e.g., Mazeh et al. 2000; Brown et al. 2001; Laughlin et al. 2005; Winn \& Holman 2005).

To date, nine additional planets have been found to transit their parent stars, and the varied uses to which these transits have been put are described in the review article by Charbonneau et al. (2006). Our goal in this paper is to ascertain whether the two outer planets orbiting Gl 876 (IL Aqr, GJ 876, HIP 113020) can similarly be observed in transit. A positive detection would represent a further major improvement in our understanding both of this specific system and of planetary properties in general.

Rivera et al. (2005) carried out a photometric search for transits by planet $\mathrm{d}(P \sim 1.974$ day $)$, and showed that such transits do not occur. To date, photometric searches for transits by planet $\mathrm{c}(P \sim 30$ day $)$ and $\mathrm{b}(P \sim 60$ day $)$ have not been reported. Although the a priori geometric transit probabilities for G1 $876 \mathrm{c}$ and $\mathrm{b}$ are only $P_{\mathrm{tr}}=1.4 \%$ and $P_{\mathrm{tr}}=0.9 \%$, respectively, the expected photometric transit depths are greater than $10 \%$. Librational motions arising from the resonance between the two outer planets lead to predicted transit epochs for the outer planets that are not 
TABLE 1

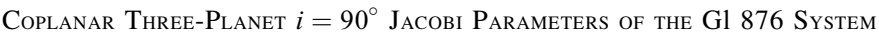

\begin{tabular}{|c|c|c|c|}
\hline Parameter & Planet d & Planet c & Planet $b$ \\
\hline 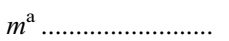 & $5.89 \pm 0.54 M_{\oplus}$ & $0.619 \pm 0.005 M_{\mathrm{Jup}}$ & $1.935 \pm 0.007 M_{\text {Jup }}$ \\
\hline 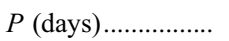 & $1.93776 \pm 0.00007$ & $30.340 \pm 0.013$ & $60.940 \pm 0.013$ \\
\hline$K\left(\mathrm{~m} \mathrm{~s}^{-1}\right) \ldots \ldots \ldots \ldots$ & $6.46 \pm 0.59$ & $88.36 \pm 0.72$ & $212.60 \pm 0.76$ \\
\hline$a^{\mathrm{a}}(\mathrm{AU}) \ldots \ldots \ldots \ldots \ldots$ & $0.0208067 \pm 0.0000005$ & $0.13030 \pm 0.00004$ & $0.20783 \pm 0.00003$ \\
\hline 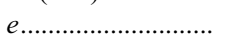 & 0 (fixed) & $0.2243 \pm 0.0013$ & $0.0249 \pm 0.0026$ \\
\hline 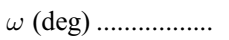 & 0 (fixed) & $198.3 \pm 0.9$ & $175.7 \pm 6.0$ \\
\hline$M(\mathrm{deg}) \ldots \ldots \ldots \ldots \ldots$ & $309.5 \pm 5.1$ & $308.5 \pm 1.4$ & $175.5 \pm 6.0$ \\
\hline transit epoch......... & JD 2,452,490.756 \pm 0.027 & JD $2,452,517.633 \pm 0.051$ & $\ldots$ \\
\hline
\end{tabular}

\footnotetext{
${ }^{a}$ Quoted uncertainties in planetary masses and semimajor axes do not incorporate the uncertainty in the mass of the star.
}

spaced evenly in time. (The resonant structure of the system is discussed further in Laughlin \& Chambers 2001; Rivera \& Lissauer 2001; Kley 2003; and Beaugé et al. 2005).

Benedict et al. (2002) used Hubble Space Telescope (HST) astrometry to derive an orbital inclination for Gl 876b of $i=$ $84^{\circ} \pm 6^{\circ}$. Their result thus suggests that planet $b$ has a mass equal or close to the radial velocity ( $\mathrm{RV})$-determined value of $M \sin (i)=$ $1.89 M_{\text {Jup }}$, and that the system is within $1 \sigma$ of being viewed edge-on. The implication of the Benedict et al. (2002) measurement is that occurrence of transits is much more likely than the a priori geometric probability would suggest. The Benedict et al. (2002) result is in conflict, however, with the dynamical models of the system presented by Rivera et al. (2005), who find a best fit to the radial velocity data when the system (assumed coplanar) is inclined by $i \sim 50^{\circ} \pm 3^{\circ}$ with respect to the plane of the sky. Therefore, if transits are detected, then the Benedict et al. (2002) results will be confirmed at the expense of the conclusions of Rivera et al. (2005).

Gl 876b and G1 876c are members of an as yet unobserved class of giant planets with intermediate surface temperatures. The expected effective temperatures of $T_{\text {eff }, \mathrm{b}}=160 \mathrm{~K}$ and $T_{\text {eff, },}=200 \mathrm{~K}$ of the planets are much lower than the value $T_{\text {eff }} \sim 1350 \mathrm{~K}$ measured with Spitzer for HD 209458b (Charbonneau et al. 2005), but still considerably higher than the $T_{\text {eff }} \sim 135 \mathrm{~K}$ measured for Jupiter. Determination of their physical properties would thus provide a useful link between Jupiter and Saturn on the one hand, and objects such as HD 209458b on the other. Accurate measurement of their radii would also give clues to their interior structure (see Charbonneau et al. 2006).

Finally, the G1 876 system with its strongly gravitationally interacting planets and large planet-to-star radius ratios, has the potential to display an extraordinarily informative set of transit light curves. Our model light curves here show what one can expect when interacting transiting systems are discovered, for example, with ongoing radial velocity surveys or with space-based missions such as Kepler (Borucki et al. 2003) or COROT (Baglin 2003), and allow for a more complete understanding of the multiplanet dynamics.

The plan of this paper is as follows. In $\S 2$ we describe our dynamical model of the system, and illustrate some of the photometric properties that the in-transit light curves would be expected to display. In $\S 3$ we describe an evaluation of the Doppler radial velocity measurements that have been taken during predicted transit intervals. In $\S 4$ we evaluate a range of archival and newly obtained photometric data sets for evidence of transits. In $\S 5$ we discuss our results, as well as the current status and outlook for the ongoing Transitsearch collaboration and conclude in $\S 6$.

\section{THE DYNAMICAL MODEL}

G1 876 (M4V) is the 40th nearest star to Earth, with a Hipparcosdetermined distance of 4.69 pc (Perryman et al. 1997); Tycho-II and UCAC positions validate this determination. As discussed in Laughlin et al. (2005), current estimates of its mass and radius stand at $M_{\star}=0.32 M_{\odot}$ and $R_{\star}=0.3 R_{\odot}$. We adopt these values as fixed in this paper. To date, 155 Doppler velocities measured at the Keck telescope and 16 velocities measured at the Lick telescope have been published for the G1 876 system (Marcy et al. 2001; Rivera et al. 2005). The two planets induce a total velocity half-amplitude of the star of nearly $0.25 \mathrm{~km} \mathrm{~s}^{-1}$. Furthermore, as discussed in Rivera et al. (2005), the system displays a very low level of stellar radial velocity "jitter," which further aids in obtaining a detailed characterization of the orbits. The system has now been observed for more than 80 orbital periods of the middle planet, $c$. This extensive time baseline allows for a much more detailed study of planet-planet interactions than can be obtained with the other exoplanetary systems known to be in 2:1 resonance, a list that now includes HD 82943 (Mayor et al. 2004; Lee et al. 2006), HD 128311 (Vogt et al. 2005), and HD 73526 (Tinney et al. 2006). These three systems all have inner planet periods on the order of 10 times longer than Gl 876c. Our baseline coplanar $i=90^{\circ}$ orbital model was obtained by Rivera et al. (2005) from a dynamical fit to the Keck radial velocity data. For reference, the parameters of this model are reproduced in Table $1 .{ }^{13}$ Additional fits with $i<90^{\circ}$ were also obtained to the Keck data using the algorithm described by Rivera et al. (2005). As with the $i=90^{\circ}$ baseline model, the system is assumed to contain three planets, all orbiting the star in the same plane. We varied the inclination of the orbital plane to the plane of the sky from $i=90^{\circ}$ to $89^{\circ} .0$ in decrements of $0^{\circ} .1$, and fitted for each of the $13+1$ remaining parameters. Then $N$-body integrations of the fit generate a sequence of transit predictions for the outer two planets. Uncertainties in the predicted transit midpoints were generated with the bootstrap Monte Carlo technique described by Rivera et al. (2005). For both outer planets, the uncertainty in the transit midpoint times was found to vary from $\sim 1$ to a few hours. The variation in uncertainty from transit to transit is plotted in Figure 1. The predicted transit midpoints are best constrained during the epoch spanned by the radial velocity observations. Errors increase as the transit predictions are generated for earlier or later times. Light curves were produced with a photometric model of the transit, which assumes that the planets are opaque disks with radii $R=0.93 R_{\text {Jup }}$

\footnotetext{
13 Note that the elements reported in Table 1 are osculating orbital elements (see, e.g., Murray \& Dermott 1999) referenced to the epoch JD 2,452,490.
} 


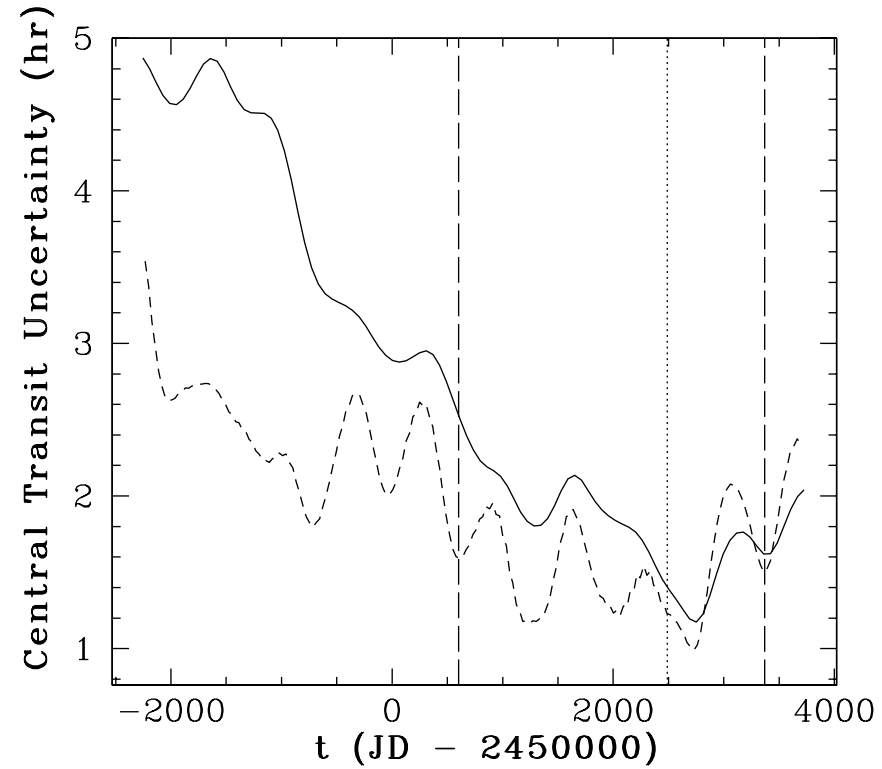

FIG. 1.-Uncertainty in the central transit time for planets G1 876b (solid line) and G1 876c (dashed line). The vertical dashed lines delineate the time span of the Keck RV observations used to generate the coplanar fits that form the basis of our model photometric and RV curves. The vertical dotted line indicates the epoch for the fits (JD 2,452,490.0).

for $\mathrm{c}$ and $R=1.04 R_{\mathrm{Jup}}$ for $\mathrm{b}$. These equatorial radii are computed using the models of Bodenheimer et al. (2003). The specific intensity of the stellar disk is modeled with a linear limb darkening law, given as

$$
I(\mu) / I(1)=1-v(1-\mu),
$$

where $I$ is the specific intensity of the stellar disk, $v=0.724$ is the corresponding limb darkening coefficient appropriate to $V$-band observations of an M4V primary (per Claret 2000), and $\mu=\cos (\theta)$.

Figures 2 and 3 show a selection of model light curves for planets $\mathrm{b}$ and $\mathrm{c}$. For planet $\mathrm{b}$, which has an eccentric $(e=0.22)$, rapidly precessing orbit $\left(d \varpi / d t \sim 44^{\circ} \mathrm{yr}^{-1}\right)$, the shape of the

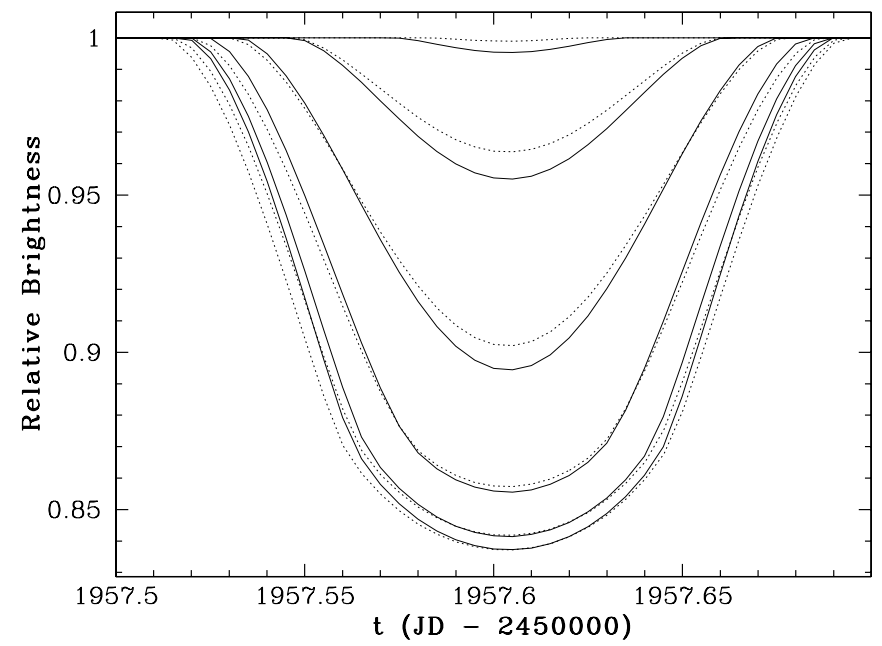

FIG. 2.-Model limb-darkened light curves during a predicted transit by planet G1 876b when it is near periastron (solid curves) and near apastron (dotted curves). The curves correspond to different assumed inclinations $\left(i=90^{\circ}, 89^{\circ} .9\right.$, $\left.89^{\circ} .8, \ldots, 89^{\circ} .5\right)$. The shallowest occur for $i=89^{\circ} .5$.

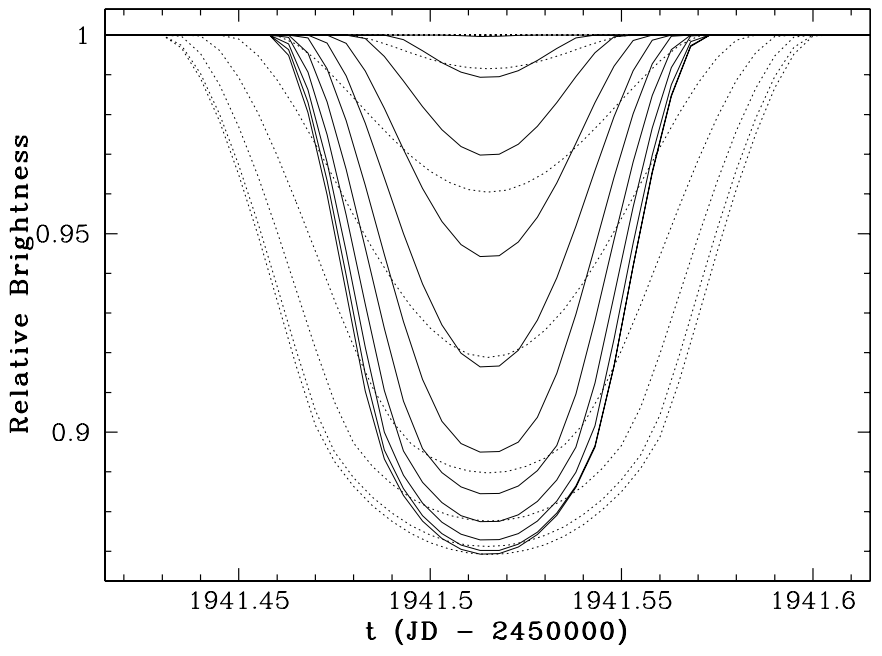

FIG. 3.-Model transit light curves for planet Gl 876c, when it is near periastron (solid curves), and near apastron (dotted curves). Inclinations, $i=90^{\circ}$ through $i=89^{\circ} .0$ are shown in decrements of $0{ }^{\circ} .1$. Planet Gl 876c's eccentricity $e \sim 0.22$ leads to a greater difference in transit depth and duration between periastron and apastron.

predicted curves depends strongly on the osculating periastron angle. For $i<90^{\circ}$, the transits are both shorter and deeper when the transits occur near periastron. The orbital eccentricity of planet $\mathrm{b}$ is more nearly circular, with $e=0.025$. In this case, there is little variation in the predicted light curves as the orbits precess. The known transiting planets have transit-to-transit intervals $\Delta T_{i}$ that are evenly spaced to within the current resolution of the observations. HD 209458b, for example, has had individual transit midpoints measured to an accuracy of several seconds (Brown et al. 2001; Wittenmyer et al. 2005), and an average period measured to an accuracy of $83 \mathrm{~ms}$ ( Wittenmyer et al. 2005; Winn \& Holman 2005). If, however, one is able to measure differences in the time intervals between successive transits, one can derive additional dynamical information. As discussed by Miralda-Escudé (2002), Holman \& Murray (2005), and Agol et al. (2005), measurements of small variations from exact periodicity can be used to infer the presence of additional bodies in the system, potentially with the masses in the range appropriate to terrestrial planets. In the event that either G1 876b or G1 876c were to transit, one would expect the departures from strict periodicity to be large. In fact, successive transits of planet $\mathrm{c}$ would occur at intervals differing by as much as $4 \mathrm{hr}$. By fitting a dynamical model to such transit intervals, one could obtain information regarding all of the orbital elements of the planets (including inclinations and nodes).

\section{SEEKING EVIDENCE OF TRANSITS IN THE RADIAL VELOCITY DATA SET}

During a planetary transit, the occulting disk of the planet passes over portions of the stellar disk containing a varying component of line-of-sight velocity arising from stellar rotation; this phenomenon produces an asymmetry in the star's spectral lines known as the Rossiter-McLaughlin effect (Rossiter 1924; McLaughlin 1924). The effect has been described in detail in the context of planetary transits by Ohta et al. (2005), and has been analyzed for HD 209458 by Queloz et al. (2000), Bundy \& Marcy (2000), and Winn et al. (2005), and for HD 149026 by Wolf et al. (2006). In fact, the transiting planet HD 189733 was initially discovered to transit not via photometry, but rather via such in-transit radial velocity variations found by Bouchy et al. (2005). For our study of G1 876, we compared the observation epochs of all of 


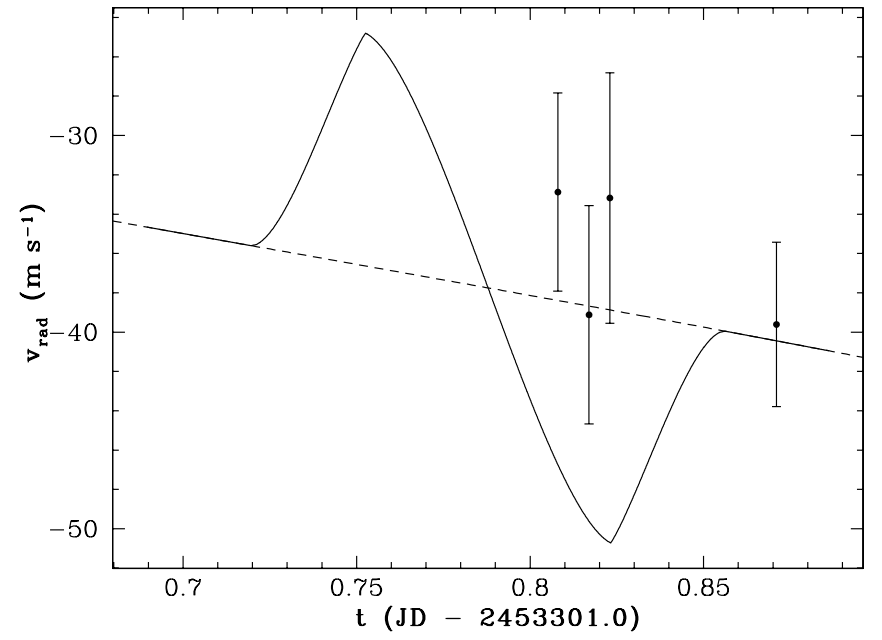

FIG. 4.- Three-planet RV model (dashed line) with a superimposed model Rossiter curve (solid line) during a predicted transit by planet G1 876c near epoch JD 2,453,301.79. The observed Keck RV data are shown as the black filled-in circles with error bars.

the Lick (see Marcy et al. 2001) and Keck (see Rivera et al. 2005) radial velocity observations to see if any of these observations occurred during a predicted transit (assuming $i=90^{\circ}$ ). For Gl 876, we used a rotational period that was determined to be 96.7 days using rotational modulation (as reported in Rivera et al. 2005); we used this value to calculate the amplitude of the RossitterMcLaughlin effect. Four such observations were found, three taken during a predicted transit by planet $\mathrm{c}$, and one taken during a predicted transit by planet $b$. Figures 4 and 5 display the relevant parts of the radial velocity model (both with and without the RossiterMcLaughlin effect), with the observations shown. The radial velocity model curves in both figures were generated by adding template curves that use the stellar and planetary radii and limb darkening law given above (and that apply the analytic integrals given in Ohta et al. 2005), to our fiducial $i=90^{\circ}$ three-planet radial velocity model. For the transit epoch in the case of planet $\mathrm{c}$ (Fig. 4), the data are inconsistent with a transit. In Figure 5, for the outer planet $b$, the situation is unresolved.

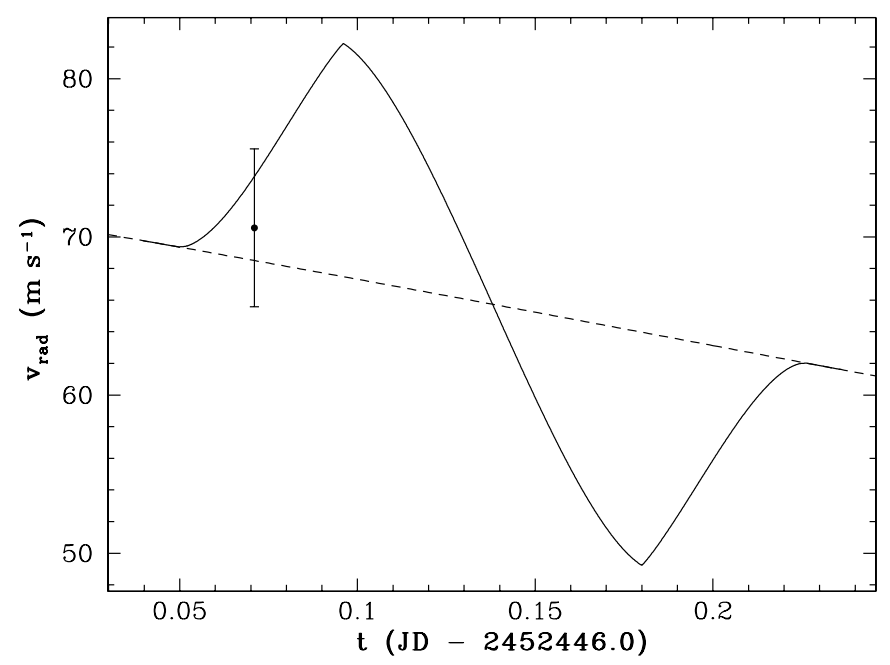

FIG. 5.-Three-planet RV model (dashed line) with a superimposed model Rossiter curve (solid line) during a predicted transit by planet G1 876b near epoch JD 2,452,446.14. Again, the observed Keck RV data are shown as the black filledin circle with error bars.

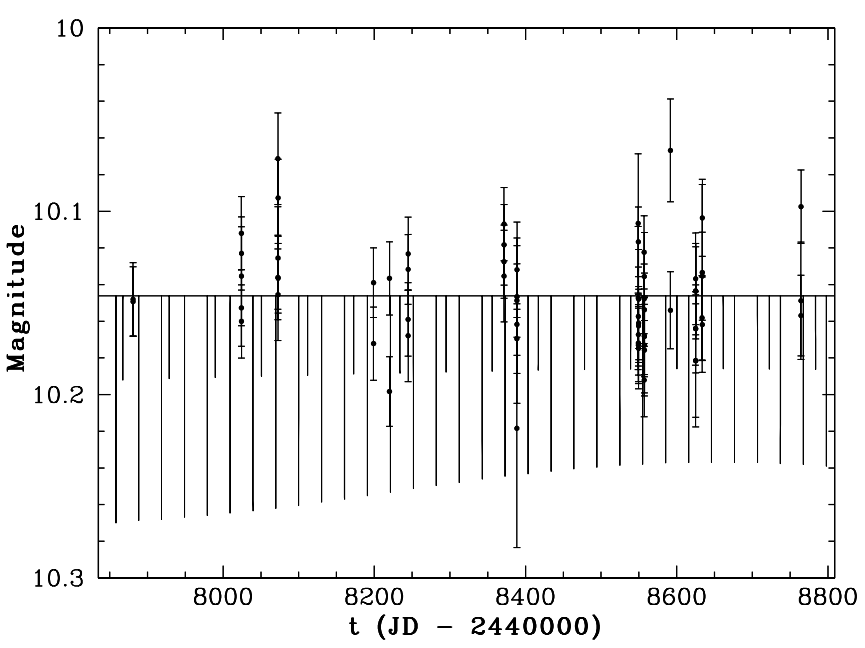

FIG. 6.- Model $i=89^{\circ} 6$ light curves for Gl $876 \mathrm{~b}$ and c superimposed on Hipparcos epoch photometry. None of the Hipparcos measurements were made near or during a predicted transit window.

\section{A SEARCH FOR TRANSITS IN PHOTOMETRIC DATA SETS}

We have also compared our model light curves to a variety of photometric data sets for G1 876 taken since 1989. The results of these comparisons are as follows.

Hipparcos. - The Hipparcos mission (see Perryman et al. 1997) produced 67 accepted photometric measurements for G1 876 (HIP 113020). These measurements have a median magnitude $H_{P}=10.148$, with $\sigma=0.0045$. There is evidence for a weak periodicity when the data is folded at the 96.7 days rotational period of the star (also noted in Rivera et al. 2005). In Figure 6, we plot our model $i=89^{\circ} .6$ light curves for $\mathrm{Gl} 876 \mathrm{~b}$ and c superimposed on the Hipparcos epoch photometry. None of the Hipparcos measurements were made near or during a predicted transit window.

Fairborn Observatory.- - A number of long-term photometric monitoring programs are being carried out with the automated telescopes at Fairborn Observatory (e.g., Henry 1999; Eaton et al. 2003) and, as described in detail in Rivera et al. (2005), Gl 876 has been on one of the observing programs. Figures 7 and 8 show the Fairborn Observatory data and our corresponding model light

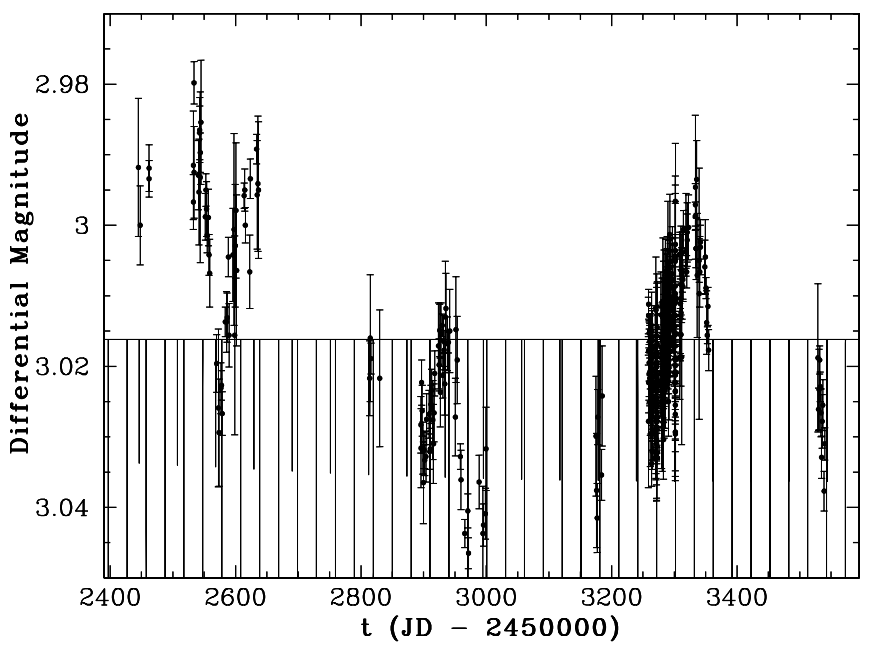

FIG. 7.- Model $i=89^{\circ} .3$ light curves for Gl $876 \mathrm{~b}$ and c superimposed on Fairborn Observatory photometry. Note that the intrinsic long-term variability for Gl 876 over a $\sim 1000$ day period is of order $0.05 \mathrm{mag}$. 


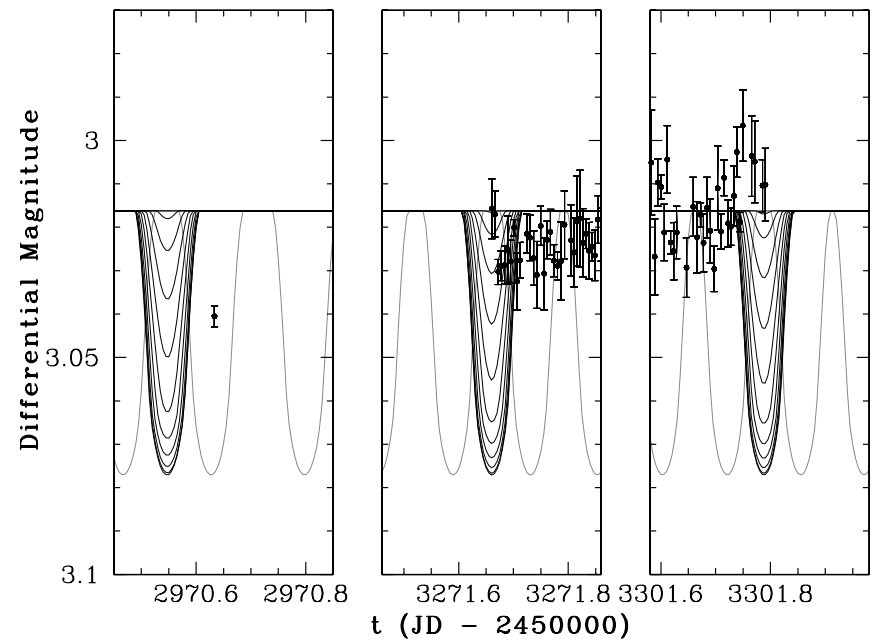

FIG. 8.- Model light curves for G1 876c superimposed on Fairborn Observatory photometry taken near three individual predicted transit windows. The solid lines correspond to model light curves arising from assumed inclinations running from $i=90^{\circ} .0$ to $i=89^{\circ}$. 0 . The light lines correspond to $i=90^{\circ}$ model light curves arising from bootstrap fits in which the transit is $3 \sigma$ early, $1 \sigma$ early, and $1 \sigma$ and $3 \sigma$ late.

curve for planets $\mathrm{b}$ and $\mathrm{c}$. Data taken during predicted transit epochs for planet c show no indication of a transit. The Fairborn Observatory data indicate that long-term stellar variability from G1 876 is of the order $0.05 \mathrm{mag}$, which is somewhat smaller than the variation observed with Hipparcos.

Transitsearch and AAVSO ${ }^{14}$.- Seagroves et al. (2003) describe Transitsearch as a cooperative distributed observing project involving submeter class telescopes worldwide. The Transitsearch strategy is to observe known planet-bearing stars at the dates and times when transits are expected to occur. It is therefore a targeted search, which differentiates it from ongoing wide-field surveys. We identify observing windows for candidate stars through the use of the bootstrap Monte Carlo technique described by Laughlin et al. (2005). The observational campaigns are pri-

14 See http://www.aavso.org. oritized by the a priori likelihood that a particular candidate planet will display transits. This likelihood is given by

$P_{\text {transit }}=0.0045\left(\frac{1 \mathrm{AU}}{a}\right)\left(\frac{R_{\star}+R_{\mathrm{pl}}}{R_{\odot}}\right)\left[\frac{1+e \cos (\pi / 2-\varpi)}{1-e^{2}}\right]$,

where $a$ is the semimajor axis of the orbit, $R_{\star}$ is the radius of the star, $R_{\mathrm{pl}}$ is the radius of the planet, $\varpi$ is the longitude of periastron, and $e$ is the orbital eccentricity.

The first Transitsearch data set for Gl 876 was obtained by one of us (P. D. S. Shankland) from 2003 October 27.9583 to October 28.0368 , with a $V$-filtered, 16-bit speckle CCD at the primary focus of a $40.4 \mathrm{~cm} \mathrm{f} / 5.1$ reflector (at air mass from $\sec z=2.7-1.7$ ). The photometric time series shows a monotonic flux increase of $0.16 \pm 0.04$ mag from 2003 October 27.9583 to October 27.9972. As shown on the left panel of Figure 9, the depth, duration, and timing of this event were consistent with an egress from transit of Gl 876c, in agreement with our predictions. The possibility that a transit had been observed spurred us to orchestrate a distributed observational campaign during the 2004 season.

During the 2004 June 24 opportunity, observations were attempted by 10 observers in Australia. Poor weather thwarted all but one observer, and no transit signal was seen in the data, which covered about half of the $1 \sigma$ transit window. Eight Australian and Japanese observers participated in the following 2004 July 24 planet c campaign. They were all clouded out. Weather improved for the 2004 August 23 planet c opportunity, permitting observations by five of the nine Australian, South African, and German observers. No transit signal was seen with photometric depth greater than $1 \%$.

In 2004 October, a number of AAVSO and Transitsearch observers obtained photometry during a closely spaced pair of transit windows for planets $\mathrm{b}$ and $\mathrm{c}$. Beginning October 20 11:22 (UT) and ending October 23 15:27 (UT), a total of 2795 CCD observations were obtained. In addition, 2981 photometric observations were obtained in the days immediately before and after the window to set baseline activity and to look for red dwarf flaring. Such a large, distributed network of observers allowed coverage of the window with very few observing gaps over the 3 day period. Gaps in the coverage were smaller than the estimated transit period or forecast window, allowing us to conclude that transits for planets
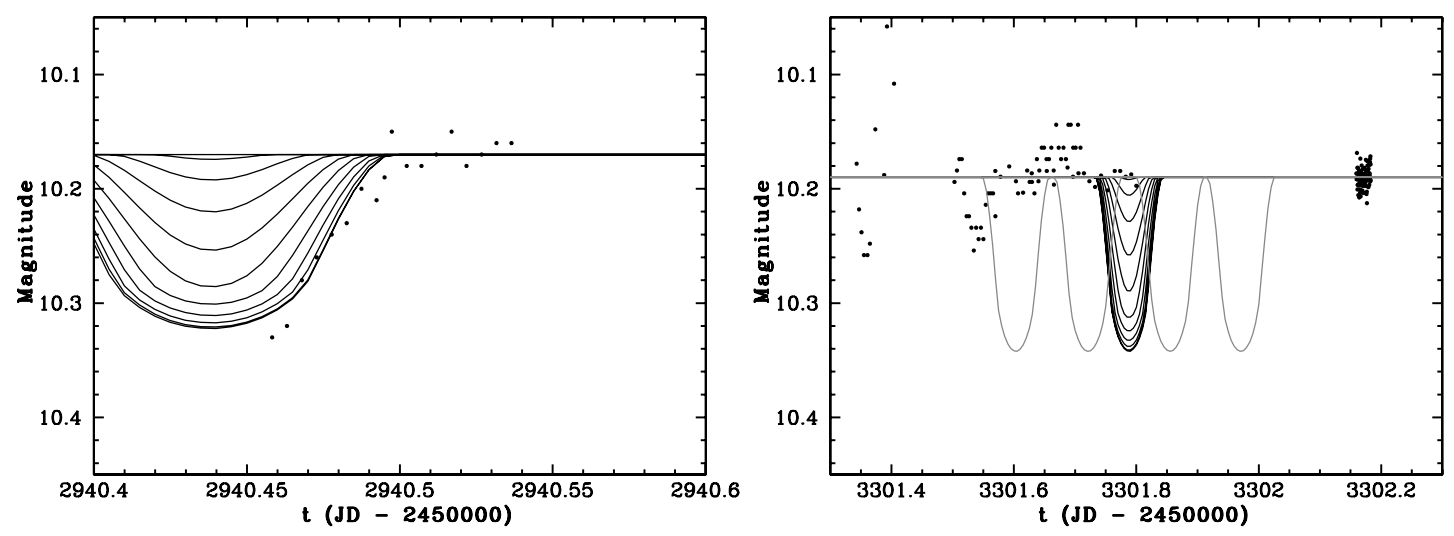

FIG. 9.-Left: data for G1876c, this plate shows dynamical model light curves again for the same decrements of $i$, taken near JD 2,452,940.4381; relative photometry,

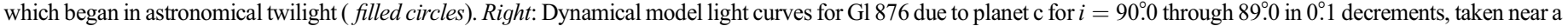

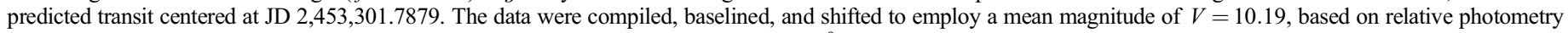

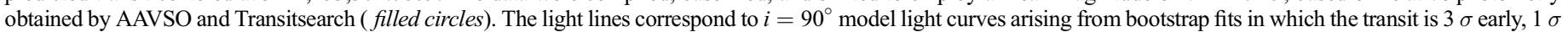
early, and $1 \sigma$ late and $3 \sigma$ late. 




FIG. 10.-Dynamical model light curves for G1 876 due to planet $\mathrm{b}$ for $i=$ $90^{\circ} .0$ through $89^{\circ} .5$ in $0^{\circ} .1$ decrements, near a predicted transit centered at JD $2,453,300.3806$. As with the previous figure, the data were baselined and shifted to employ a mean magnitude of $V=10.19$, based on relative photometry obtained by AAVSO and Transitsearch (filled circles). The light lines correspond to $i=90^{\circ}$ model light curves arising from bootstrap fits in which the transit is $3 \sigma$ early, $1 \sigma$ early, and $1 \sigma$ late and $3 \sigma$ late.

$\mathrm{b}$ and $\mathrm{c}$ did not occur during the 2004 October campaign. Photometric data from the campaign are plotted in Figures 9 and 10.

Individual observations and uncertainty estimates from the 2004 October campaign are available from the AAVSO Web site, while the other data sets can be found at the Transitsearch website. ${ }^{15}$ We are continuing to obtain photometry of Gl 876 , with the goal of gaining a better understanding of the photometric variability of mature (age exceeding 1 Gyr) red dwarfs.

\section{DISCUSSION}

The Transitsearch photometric network has been in operation for $3 \mathrm{yr}$, and has participating observers capable of providing fully global longitude and latitude coverage. The network maintains a continuously updated catalog ${ }^{16}$ of the known census of extrasolar planets. The catalog is currently the only available source for (1) a priori transit probabilities and predicted transit depths for the known extrasolar planets, (2) predicted transit ephemeris and transit window uncertainties based on orbital fits to published radial velocities, and (3) the results of known photometric searches for transits of planet-bearing stars. To date, the majority of negative transit searches have not been reported in the literature. With this paper, we hope to spur a reversal of that trend.

Due to significant uncertainties in the orbital fits, it is generally very difficult to definitively rule out transits by planets with $P>$ 10 day. The Gl 876 planets form a notable exception to this rule because of their high signal-to-noise ratio $\Gamma \sim 50$, and the extensive radial velocity data set allows for a very accurate orbital fit. Campaigns by the Transitsearch collaboration have thus far led to transits of a number of other shorter-period planet-bearing stars being ruled out to a high degree of confidence. The planets for which the possibility of transits have been significantly discounted by our campaigns include HD $217107 \mathrm{~b}\left(P=7.127\right.$ day, $P_{\mathrm{tr}}=$ $8.0 \%$; see also Vogt et al. 2005), HD $168746 \mathrm{~b}(P=6.403$ day,

\footnotetext{
15 Our AAVSO data are available at http://www.aavso.org/data/download/, while other collected data can be found at http://www.ucolick.org/\%7elaugh/ G1876/_c.results.html.

16 Available at http://www.ucolick.org/\%7elaugh/.
}

$P_{\mathrm{tr}}=8.1 \%$; see also Pepe et al. 2002), and HD 68988b $(P=$ 6.276 day, $P_{\mathrm{tr}}=9.0 \%$; see also Vogt et al. 2002).

Furthermore, planets for which usable photometry of the parent stars has been obtained by our network during the $3 \sigma$ transit windows, but for which transits cannot yet be fully ruled out, include HD $188753 \mathrm{Ab}\left(P=3.348\right.$ day, $\left.P_{\mathrm{tr}}=11.8 \%\right)$, HD $76700 \mathrm{~b}(P=$ 3.971 day, $\left.P_{\mathrm{tr}}=10.0 \%\right)$, HD $13445 \mathrm{~b}\left(P=15.76\right.$ day, $P_{\mathrm{tr}}=$ $3.4 \%)$, HD $74156 \mathrm{~b}\left(P=51.64\right.$ day, $\left.P_{\mathrm{tr}}=3.8 \%\right)$, HD $37605 \mathrm{~b}$ $\left(P=54.23\right.$ day, $\left.P_{\mathrm{tr}}=2.2 \%\right)$, and HD $80606 \mathrm{~b}(P=111.4$ day, $\left.P_{\mathrm{tr}}=1.7 \%\right)$. Planets for which campaigns are scheduled to begin soon include GL 581b $\left(P=5.366\right.$ day, $\left.P_{\mathrm{tr}}=3.6 \%\right)$, and HD 99492b $\left(P=17.04\right.$ day, $\left.P_{\mathrm{tr}}=3.4 \%\right)$. GL 581 is of particular interest because the primary star is an $\mathrm{M}$ dwarf, and the planet (if it transits) will be a Neptune-mass object $M \sin i=0.052 M_{\text {Jup }}$ (Bonfils et al. 2005). The 1.6\% transit depth would allow an unprecedented physical characterization of a low-mass extrasolar planet. To date, our Gl 876 campaign has employed the most globally far-reaching distribution, and along with recent leaps in fidelity in similar campaigns, clearly underscores the utility of the network.

What is more, G1 $876 \mathrm{~b}$ and c constitute a dramatic exception to the emerging aphorism that Jupiter-mass planets are rarely associated with $\mathrm{M}$ dwarfs. Indeed, a paucity of giant planets orbiting red dwarfs seems to be a natural consequence of the core accretion theory of planet formation (Laughlin et al. 2004). The coreaccretion theory predicts, however, that Neptune-mass and smaller mass planets will be very common around red dwarfs. This stands in contrast to the predictions of Boss (2006), who suggests that the gravitational instability mechanism is the dominant mode of giant planet formation. If this is true, then Jupiter-mass planets should be just as common around red dwarfs as they are around solar-type stars. Efforts to detect transits of small planets orbiting small stars are therefore likely to gain increasing importance and focus over the next several years.

There are, in fact, several observational indications that lowmass planets may be common around red dwarfs. Radial velocity surveys have recently reported the detection of Neptune-mass companions in short-period orbits around the red dwarfs GL 581 and GL 436. The Optical Gravitational Lensing Experiment (OGLE) team of observers using the microlensing method have detected the signature of what appears to be a planet with $5.5 M_{\oplus}$ orbiting a distant red dwarf (Beaulieu et al. 2006). With these points in mind, we can put the results of our Gl 876 campaign in a broader context. A ground-based (and "fiscally viable") photometric network such as Transitsearch, especially in collaboration with a dedicated bank of telescopes, can capably monitor individual $\mathrm{M}$ dwarfs to search for transits of terrestrial-sized planets. Owing to the intrinsic long-term variability of $\mathrm{M}$ dwarfs, candidate planets would have to be identified on the basis of a full transit signature in a single time series.

A $0.1 M_{\odot} \mathrm{M}$ dwarf has $R_{\star} / R_{\odot} \sim 0.1, T_{\text {eff }} \sim 2750 \mathrm{~K}$, and $L_{\star} / L_{\odot} \sim 5 \times 10^{-5}$; a habitable planet that receives an Earthequivalent flux from the star thus needs to orbit at a distance of just $0.022 \mathrm{AU}$, which corresponds to an orbital period of 3.85 days. An Earth-like planet with such a period would be rotationally synchronized to the red dwarf, and in the absence of any significant perturbing bodies, its orbit would be almost perfectly circular. Interestingly, however, simulations show that habitability is unlikely to be adversely affected by a spin-orbit period synchronization. Joshi et al. (1997) used a global climate model to investigate how the Earth's climate would respond if the Earth were tidally locked to the Sun, and found that Earth remains habitable in this configuration, at $1 \mathrm{AU}$, and perhaps throughout the habitable zone. 
The $M=7.5 M_{\oplus}, P=1.9379$ day companion to Gl 876 demonstrates that it is not unreasonable to expect terrestrial-mass bodies on short-period orbits of $0.1 M_{\odot}$ stars. R. Montgomery \& G. Laughlin (private communication, 2006) have carried out accretion simulations with the Wetherill-Chambers method (Wetherill 1996; Chambers 2001) that model the accretion of terrestrial-mass planets in short-period orbits about M dwarfs. These simulations provide further evidence that terrestrial-mass planets will commonly form in the desired short-period orbits.

If habitable planets do commonly form in orbit around low mass $\mathrm{M}$ dwarfs, the chances of detecting and characterizing them are surprisingly good. The transit of an Earth-sized planet will block about $1 \%$ of the stellar flux of a $0.1 M_{\oplus}$ star. For a planet on a habitable 3.85 day orbit, the transit will be relatively brief, $\sim 40$ minutes. The a priori geometric probability of observing such a transit is $2 \%$.

A $1 \%$ photometric dip is readily detectable. Amateur astronomers who participate in the Transitsearch collaboration routinely achieve detection thresholds of considerably better than $1 \%$, as evidenced by confirming detections of HD 149026b, which has a transit depth of just $0.3 \%$. Indeed, capable amateur observers have demonstrated the photometric capability to detect the passage of a Mars-sized body in front of an 11th mag $0.1 M_{\odot}$ red dwarf.

Inevitably one must ask how many suitable red dwarfs are available on the sky as a whole. Even though the lowest mass $\mathrm{M}$ stars are the most common type of star, they are also exceedingly $\operatorname{dim}$. A dedicated $3 \mathrm{~m}$ class telescope would be required to properly search the magnitude range $V=16-18$, where observations of the lowest mass red dwarfs begin to be plentiful (and indeed, many $0.1 M_{\odot}$ stars within 10 pc still likely remain to be discovered). In the meantime, however, we recommend that observers obtain high-cadence photometry of nearby single stars listed in the RECONS catalog 17 of the 100 nearest stellar systems; these include Proxima Centauri, Barnard's Star, Wolf 359, Ross 154, Ross 128, DX Cancri, GJ 1061, GJ 54.1, and GJ 83.1. We estimate that each one has a $\sim 1 \%$ chance of harboring a detectable transiting, potentially habitable planet, readily detectable by distributed photometric observation.

${ }^{17}$ Maintained by T. Henry and collaborators at Georgia State University.

\section{CONCLUSIONS}

Our study of G1 876 has produced several interesting results. We have demonstrated that transits of planets $b$ and $c$ are not currently occurring, and we have thus modestly constrained the parameter space available to observationally allowed orbital configurations of the G1 876 system. The absence of transits is consistent with the dynamical study of Rivera et al. (2005), who find that a coplanar system inclination $i \sim 50^{\circ}$ yields the best fit to the radial velocity data. Our dynamical analysis indicates that when planetary transits in a strongly interacting system similar to Gl 876 are eventually observed, then a tremendous amount of information will be obtained. We look forward to the discovery of the first such system.

We have also shown that a globally distributed network of smalltelescope observers can effectively provide definitive photometric follow-up of known planet-bearing low-mass stars. Our experience with Gl 876 indicates that intensive photometric monitoring campaigns of individual, nearby $\mathrm{M}$ dwarfs constitute a tractable strategy for planet detection. The Sun's nearest low-mass stellar neighbors should therefore be scrutinized very carefully for transits by short-period terrestrial planets. A low-budget survey of this type provides a small, but nevertheless nonnegligible chance to discover a truly habitable world from the ground and within a year or two. Such a detection would be a remarkable and exciting discovery.

We thank K. Johnston, R. Gaume, K. Malatesta, J. Lazio, D. Boboltz, C. Shaw, B. Casey, E. Albert, A. Henden, J. Larsen, D. Katz, C. Phillips, W. Bollwerk, Z. Dugan, J. Jaglowicz, and T. Castellano for useful discussions or other support. We are grateful to the AAVSO and its many members who provided campaign support in addition to those authors listed above. We also gratefully acknowledge the insightful suggestions and constructive feedback offered by our referee. This material is based on work supported by the National Aeronautics and Space Administration under grant NNG04GN30G issued through the Origins of Solar Systems Program to G.L. G. W. H. acknowledges support from NASA grant NCC5-511 and NSF grant HRD-9550561. This research has made use of the Simbad, USNO, and transitsearch databases, operated at CDS, Strasbourg, France, USNO, Washington, DC, and UCSC, Santa Cruz, California, respectively.
Agol, E., Steffen, J., Sari, R., \& Clarkson, W. 2005, MNRAS, 359, 567

Baglin, A. 2003, Adv. Space Res., 31, 345

Beaugé, C., Michtchenko, T., \& Ferraz-Mello, S. 2006, MNRAS, 365, 1160

Beaulieu, J.-P., et al. 2006, Nature, 439, 437

Benedict, G. F., et al. 2002, ApJ, 581, L115

Bodenheimer, P., Laughlin, G., \& Lin, D. N. C. 2003, ApJ, 592, 555

Bonfils, X., et al. 2005, A\&A, 443, L15

Borucki, W. J., et al. 2003, in ASP Conf. Ser. 294, Scientific Frontiers in Research on Extrasolar Planets, ed. D. Deming, \& S. Seager (San Francisco: ASP), 427

Boss, A. P. 2006, ApJ, 643, 501

Bouchy, F., et al. 2005, A\&A, 444, L15

Brown, T., Charbonneau, D., Gilliland, R., Noyes, R., \& Burrows, A. 2001, ApJ, 552, 699

Bundy, K. A., \& Marcy, G. W. 2000, PASP, 112, 1421

Butler, R. P., Marcy, G. W., Williams, E., McCarthy, C., \& Vogt, S. S. 1996, PASP, 108, 500

Butler, R. P., Tinney, C. G., Marcy, G. W., Jones, H. R. A., Penny, A., \& Apps, K. 2001, ApJ, 555, 410

Chambers, J. E. 2001, Icarus, 152, 205

Charbonneau, D., Brown, T., Latham, D., \& Mayor, M. 2000, ApJ, 529, L45

Charbonneau, D., Brown, T. M., Burrows, A., \& Laughlin, G. 2006, preprint (astro-ph/0603376)

Charbonneau, D., et al. 2005, ApJ, 626, 523

\section{REFERENCES}

Claret, A. 2000, A\&A, 363, 1081

Delfosse, X., Forveille, T., Mayor, M., Perrier, C., Naef, D., \& Queloz, D. 1998, A\&A, 338, L67

Eaton, J. A., Henry, G. W., \& Fekel, F. C. 2003, The Future of Small Telescopes in the New Millennium, ed. T. D. Oswalt (Dordrecht: Kluwer)

Henry, G., Marcy, G., Butler, R., \& Vogt, S. 1999, IAU Circ., 7307, 1

. 2000, ApJ, 529, L41

Henry, G. W. 1999, PASP, 111, 845

Holman, M., \& Murray, N. 2005, Science, 307, 1288

Joshi, M. M., Haberle, R. M., \& Reynolds, R. T. 1997, Icarus, 129, 450

Kley, W. 2003, Celest. Mech. Dyn. Astron., 87, 85

Laughlin, G., Bodenheimer, P., \& Adams, F. C. 2004, ApJ, 612, L73

Laughlin, G., Butler, R. P., Fischer, D. A., Marcy, G. W., Vogt, S. S., \& Wolf, A. S. 2005, ApJ, 622, 1182

Laughlin, G., \& Chambers, J. E. 2001, ApJ, 551, L109

Lee, M. H., Butler, P., Fischer, D., Marcy, G, \& Vogt, S. 2006, ApJ, 641, 1178 Lee, M. H., \& Peale, S. J. 2002, ApJ, 567, 596

Lovis, C., et al. 2006, Nature, 441, 305

Marcy, G. W., \& Butler, R. P. 1998, ARA\&A, 36, 57

Marcy, G. W., Butler, R. P., Fischer, D., Vogt, S. S., Lissauer, J. J., \& Rivera, E. J. 2001, ApJ, 556, 296

Marcy, G. W., Butler, R. P., Fischer, D., Vogt, S. S., Wright, J. T., Tinney, C. G., \& Jones, H. R. A. 2005, Prog. Theor. Phys. Suppl., 158, 24 
Marcy, G. W., Butler, R. P., Vogt, S. S., Fischer, D. A., \& Lissauer, J. J. 1998, ApJ, 505, L147

Marcy, G. W., Cochran, W. D., \& Mayor, M. 2000, in Protostars and Planets IV, ed. V. Mannings, A. P. Boss, \& S. S. Russell (Tucson: Univ. Arizona Press)

Mayor, M., Udry, S., Naef, D., Pepe, F., Queloz, D., Santos, N. C., \& Burnet, M. 2004, A\&A 415, 391

Mazeh, T., et al. 2000, ApJ, 532, L55

McLaughlin, D. B. 1924, ApJ, 60, 22

Miralda-Escudé, J. 2002, ApJ, 564, 1019

Murray, C. D., \& Dermott, S. F. 1999, Solar System Dynamics (Cambridge: Cambridge Univ. Press)

Ohta, Y., Taruya, A., \& Suto, Y. 2005, ApJ, 622, 1118

Pepe, F., Mayor, M., Galland, F., Naef, D., Queloz, D., Santos, N. C., Udry, S., \& Burnet, M. 2002, A\&A, 388, 632

Perryman, M. A. C., et al. 1997, A\&A, 323, L49

Queloz, D., Eggenberger, A., Mayor, M., Perrier, C., Beuzit, J. L., Naef, D., Sivan, J. P., \& Udry, S. 2000, A\&A, 359, L13
Rivera, E. J., \& Lissauer, J. J. 2001, ApJ, 558, 392

Rivera, E. J., et al. 2005, ApJ, 634, 625

Rossiter, R. A. 1924, ApJ, 60, 15

Seagroves, S., Harker, J., Laughlin, G., Lacy, J., \& Castellano, T. 2003, PASP, 115,1355

Tinney, C. G., et al. 2006, ApJ, 647, 594

Vogt, S. S., Butler, R. P., Marcy, G. W., Fischer, D. A., Henry, G. W., Laughlin, G., Wright, J. T., \& Johnson, J. A. 2005, ApJ, 632, 638

Vogt, S. S., Butler, R. P., Marcy, G. W., Fischer, D. A., Pourbaix, D. Apps, K., \& Laughlin, G. 2002, ApJ, 568, 352

Wetherill, G. W. 1996, Icarus, 119, 219

Winn, J. N., \& Holman, M. J. 2005, ApJ, 628, L159

Winn, J. N., et al. 2005, ApJ, 631, 1215

Wittenmyer, R. A., et al. 2005, ApJ, 632, 1157

Wolf, A. S., Laughlin, G., Henry, G. W., Fischer, D. A., Marcy, G., Butler, P., \& Vogt, S. 2006, ApJ, in press 